\title{
CORONA (COre ultRasOund of Covid in iNtensive Care \& Acute Medicine) Study: National Service Evaluation of Lung and Heart Ultrasound in Intensive Care Patients With Suspected or Proven COVID-19
}

Prashant Parulekar ( $\sim$ Prashant.Parulekar@gmail.com )

East Kent Hospitals https://orcid.org/0000-0003-2368-5244

James Powys-Lybbe

St George's Hospital https://orcid.org/0000-0001-9348-2819

Thomas Knight

Sandwell and West Birmingham Hospitals NHS Trust

Nicholas Smallwood

Surrey and Sussex Healthcare NHS Trust

Daniel Lasserson

Sandwell and West Birmingham Hospitals NHS Trust

Gavin Rudge

University of Birmingham

Ashley Miller

Shrewsbury and Telford Hospital NHS Trust

Marcus Peck

Frimley Park Hospital NHS Foundation Trust: Frimley Health NHS Foundation Trust

Jonathon Aron

St George's Hospital

\section{Research}

Keywords: Lung Ultrasound, Echocardiography, Coronavirus, ICU

Posted Date: August 31st, 2021

DOI: https://doi.org/10.21203/rs.3.rs-824190/v1

License: (c) (1) This work is licensed under a Creative Commons Attribution 4.0 International License.

Read Full License 
Version of Record: A version of this preprint was published at Journal of the Intensive Care Society on January 10th, 2022. See the published version at https://doi.org/10.1177/17511437211065611. 


\section{Abstract \\ Background:}

Combined Lung Ultrasound (LUS) and Focused UltraSound for Intensive Care heart (FUSIC heart formerly Focused Intensive Care Echocardiography, FICE) can aid diagnosis, risk stratification and management in COVID-19. However, data on its application and results are limited to small studies in varying countries and hospitals. This United Kingdom (UK) national service evaluation study assessed how combined LUS and FUSIC Heart were used in COVID-19 Intensive Care Unit (ICU) patients during the first wave of the pandemic.

\section{Method:}

12 trusts across the UK registered for this prospective study. LUS and FUSIC Heart data were obtained, using a standardised data set including scoring of abnormalities, between 1st of February 2020 to the 30th July 2020. The scans were performed by intensivists with FUSIC Lung and Heart competency as a minimum standard. Data was anonymised locally prior to transfer to a central database.

\section{Results:}

372 studies were performed on 265 patients. There was a small but significant relationship between LUS score $>8$ and 30-day mortality (OR 1.8). Progression of score was associated with an increase in 30-day mortality (OR 1.2). 30-day mortality was increased in patients with right ventricular (RV) dysfunction $(49.4 \%$ vs $29.2 \%)$. Severity of LUS score correlated with RV dysfunction $(p<0.05)$. Change in management occurred in $65 \%$ of patients following a combined scan.

\section{Conclusions:}

In COVID-19 patients there is an association between lung ultrasound score severity, RV dysfunction and mortality identifiable by combined LUS and FUSIC Heart. Our findings suggest that serial combined LUS and FUSIC Heart on COVID-19 ICU patients may aid in clinical decision making and prognostication.

\section{Introduction}

Coronavirus disease 2019 (COVID-19) is a respiratory tract infection caused by a novel coronavirus: SARS-CoV-2. It is a heterogeneous disease with a wide spectrum of severity and organ dysfunction. In the UK, during the first wave of the pandemic, approximately $20 \%$ of hospitalised patients required admission to High Dependency Units (HDU) or ICUs ${ }^{1}$. 
Point of Care UltraSound (PoCUS) typically combines lung and cardiac imaging to obtain goal-oriented information and answer specific clinical questions. It is a non-invasive, non-radioactive, bedside modality of imaging ${ }^{2}$. In patients with COVID-19, PoCUS can aid diagnosis, risk-stratification and management decisions ${ }^{3}$. However, uncertainty remains about how to utilise PoCUS optimally.

LUS uses pleural surface changes and structural patterns of the lung to identify disease. LUS has a sensitivity between $90-100 \%$ for diagnosing infection, effusion, pneumothorax, or embolic phenomena ${ }^{4,5}$. Algorithms such as the BLUE protocol ${ }^{6}$ provide accessible methods for interpreting findings and differentiating causes of respiratory failure.

Critical care echocardiography (CCE) can be a focused or advanced structural assessment, with the option of dynamic cardiac output monitoring. CCE has become a well-established adjunct to care for initial and serial assessments of the shocked patient ${ }^{7}$. Whilst CCE is operator-dependent, focused echocardiography can provide very good correlation with departmental echocardiography - achieving a sensitivity of 0.91 and specificity of 0.88 for determining any degree of left ventricular (LV) systolic impairment ${ }^{8}$.

In patients with acute respiratory distress syndrome (ARDS), CCE features of acute cor pulmonale (ACP) are found in $20-25 \%^{9,10}$. These include RV dilation, RV impairment, septal dyskinesia and pulmonary hypertension. Lung inflammation, hypoxic vasoconstriction and high positive end-expiratory pressure (PEEP) strategies are principal in the pathology. Once established, ACP is an independent risk factor for 28-day mortality: $60 \%$ (with ACP) vs $36 \%$ (without) in patients with moderate to severe ARDS ${ }^{11}$.

As COVID-19 spread towards the UK, observational data from China ${ }^{12}$ and Italy ${ }^{13}$ supported LUS similarities between ARDS and COVID-19 pneumonitis (thickened pleura, multiple or confluent B-Lines and consolidations) in spite of two clearly different phenotypes (compliant and noncompliant ${ }^{14}$ ). Scoring mechanisms were proposed that could help enable COVID-19 diagnosis and scale illness severity ${ }^{15}$, potentially reducing the burden of pandemic on hospital resources. ${ }^{16}$

Few studies have reported focused CCE alongside LUS in COVID-1917, but echocardiographic findings correlate with changes seen in ARDS, as well as case reports of acute pulmonary embolism, dilated cardiomyopathy and acute myocardial injury ${ }^{18}$. Across 74 patients with COVID-19, one study showed RV dilatation in $41 \%$ and RV dysfunction in $27 \%$, whilst LV function was normal in $89 \%{ }^{19}$.

This paper is a nationwide service evaluation looking at the application and results of LUS and FUSIC Heart in combination during the first wave of COVID-19. This is a novel approach to the assessment of an ad hoc service managing a large number of patients with similar physiological needs. Data from ICUs within 12 registered trusts was analysed looking for observable trends that can help establish better national PoCUS practice and guidelines ${ }^{20}$. 
This paper describes findings from the intensive care arm of the CORONA study, a study jointly led by the Intensive Care Society and the Society of Acute Medicine.

\section{Methods}

A prospective service evaluation of PoCUS was completed between the $1^{\text {st }}$ February to $30^{\text {th }}$ July 2020 . Service evaluation methodology was employed to define and describe variations in approach and evaluate relationships with outcomes during routine clinical practice. All adult patients (aged $\geq 16$ years) presenting to participating hospitals with confirmed or suspected COVID-19 were eligible for inclusion. All point-of-care LUS and FUSIC Heart images obtained during acute care admission were eligible for inclusion. The analysis presented is a subset of the national evaluation for patients admitted to the ICU of each registered trust.

Demographic and clinical data were recorded using a standard proforma. All point-of-care imaging was undertaken by trained practitioners: FAMUS (Focused Acute Medicine Ultrasound) or FUSIC Lung and Heart or a higher qualification for transthoracic echocardiography. The decision to undertake LUS and the approach to imaging was determined by the treating clinician. The ultrasound device used was

determined by local availability. Serial images on the same patient were identified using a unique study ID number.

A data capture tool based on standardised point-of-care imaging protocols was provided.

LUS findings could be recorded in a maximum of 14 predefined anatomical zones. Clinicians recorded whether a 6-point, 12-point or 14-point approach had been undertaken. A semi-quantitative severity score was recorded at each anatomical location.

- Score 0: A-lines or < 2 B lines (normal aeration)

- Score 1: $\geq 2$ well-spaced B-lines (moderate loss of aeration)

- Score 2: Coalescent B lines $+/$ - small consolidations $<1 \mathrm{~cm}$ (severe loss of aeration)

- Score 3: Tissue like pattern +/- frank consolidation +/- small consolidations $>1 \mathrm{~cm}$ (complete loss of aeration)

Summated LUS severity score was approach dependent - in the case of 6-point imaging it was between 018. Presence of pleural effusion was recorded, and a subjective assessment of the pleura for each lung recorded as normal or abnormal.

Bedside transthoracic echocardiography data collection tool was derived from the FUSIC Heart protocol. This involved visual assessment for LV and RV dilatation and function. The presence of ventricular dilatation was recorded as a binary variable. Ventricular dysfunction was defined as either a dilated or visually impaired left or right ventricle. Advanced, quantitative measurements of dilatation and functional impairment were recorded in some studies. 
The indication for imaging and any immediate change of management was recorded. Patient outcome was recorded at 30-days after the imaging study.

\section{Statistics}

Analysis was performed using the R statistical software. Normally distributed continuous variables are described using mean and standard deviation and non-normally distributed continuous variables are described using median value and interquartile range. Count data is described as proportions with $95 \%$ confidence intervals.

\section{Results}

ICUs from 12 U.K. hospitals recorded 372 imaging studies in the database between $1^{\text {st }}$ February 2020 and $30^{\text {th }}$ July 2020. The median number of studies contributed by individual hospitals was 14 (IQR $3-40$ ). A total of 372 imaging studies were recorded from 265 ICU patients, 72 (27.2\%) patients had two studies and 35 (13.5\%) patients had more than two studies recorded. Paired thoracic imaging and echocardiography were recorded in 333 (89.5\%) studies. Isolated echocardiography in 32 (8.6\%) and isolated thoracic imaging was undertaken in 7 (1.9\%) studies. The median interval between ICU admission and the first imaging study was 2 days (IQR 0-8).

The mean patient age was 54 years (12.4) and 187 (70.6\%) of patients were male. The SARS-CoV-2 nasopharyngeal PCR was positive in $219(82.6 \%, 95 \% \mathrm{Cl} 77.6-86.7)$ patients at the point of the initial imaging study. The SARS-CoV-2 PCR status of 15 (5.7\%) patients was unknown.

Invasive mechanical ventilation (IMV) was being provided to 320 (86.0\%) patients at the time of imaging. Non-invasive respiratory support was being provided to $24(6.5 \%)$ patients at the time of imaging (Continuous positive airway pressure $=14$, high flow nasal support $=5$, and bi-level positive airway pressure support $=5)$. Death within 30 days of imaging occurred in $37(32.8 \% 95 \% \mathrm{Cl} 27.5-38.7)$ patients. The outcome at 30 days was not recorded for 15 (5.7\%) patients.

Thoracic imaging studies were conducted using a 6-point protocol in 305 (89.7\%) patients and a 12-point protocol in 35 (10.3\%) patients. Missing values in relation to one or more or the 6 anatomical thoracic imaging locations occurred in 17 (5.0\%) studies. A single value was missing in 15 (4.4\%) studies, two values in $1(0.3 \%)$ study and three values in 1 study $(0.3 \%)$.

The distribution of 6-point scores stratified by the interval between admission and imaging is provided in Figure 1A. The distribution of scores was asymmetric with skew towards higher scores. The distribution of scores was broadly similar when stratified by the interval between ICU admission and scan. The median score using the 6-point protocol was 11 (8-13 IQR). Ultrasound severity scores obtained utilising a 12-point protocol were compared to the 6-point score derived from the same study. The studies showed strong correlation $(0.93, \mathrm{p}$ value $=<0.001)$, (Figure $1 \mathrm{C})$. Pleural abnormalities were detected in 306 (90.0\%) images, abnormalities were unilateral in 19 (5.6\%) studies and bilateral in 287 (84.4\%) studies. 
Unilateral pleural effusions were detected in 29 (8.5\%) and bilateral pleural effusions were detected in 14 (4.1\%) studies. Of these patients, $5(35.7 \%, 95 \% \mathrm{Cl} 16.3-61.2)$ had simultaneous evidence of LV impairment. Preserved A lines in all 6 anatomical zones were observed in $9(2.6 \%)$ studies of which 7 (77.8\%) were negative for SARS-CoV-2 by PCR.

\section{Indications for scan}

The indication and outcome of studies were recorded in the database. The most common reason for undertaking imaging was for routine surveillance accounting for 164 studies (44.3\% 95\% Cl 39.3-49.4). The indication for imaging and associated distribution of 6-point lung ultrasound scores associated with each group is provided in Figure 2 and the outcome of scans is provided in Figure 3. Assessment of extravascular lung water (EVLW) was quoted as the indication for imaging in $116(31.4 \%, 95 \% \mathrm{Cl} 26.8-$ 36.2) studies. Imaging was employed to aid decision making in relation to ventilation strategy in 146 (39.5\%, 95\% Cl 34.6-44.5) studies.

\section{Lung involvement and mortality}

There was a statistically significant association between the severity of lung involvement (as assessed by 6-point severity score within 7 days of admission) and 30-day mortality (unadjusted OR 1.1 95\% $\mathrm{Cl}$ 1.01.2). This relationship remained statistically significant in a multivariate model adjusted for age, gender, interval between ICU admission and scan, and detection of SARS-CoV-2 by PCR (adjusted OR $1.195 \% \mathrm{Cl}$ $1.0-1.2$ ). The discriminatory performance of the ultrasound severity score to predict 30 -day mortality was poor (AUC 0.58).

The observed 30-day mortality associated with quartiles of lung severity score stratified by the time interval between ICU admission and the initial thoracic imaging study is shown in Figure 4A.

\section{Echocardiography}

Echocardiographic studies were recorded from 241 individual patients, of which 174 (72.8\%) were focused studies and 67 (27.2\%) were advanced bedside studies. RV dysfunction was identified in 83 $(37.7 \%, 95 \% \mathrm{Cl} 31.5-44.3)$ of the initial studies. RV function was not recorded in 6 studies (2.5\%). Observed 30-day mortality was significantly higher in patients with RV dysfunction compared with patients with normal RV function $(49.4 \% 95 \% \mathrm{Cl} 38.9-59.9$ vs, $29.2 \% 95 \% \mathrm{Cl} 22.2-37.3)$ Figure 4B. There was no significant difference in the prevalence of RV dysfunction comparing focused echocardiographic studies with advanced bedside studies $(35.1 \%, 95 \% \mathrm{Cl} 29.4-41.3$ vs $36.7 \%, 95 \% \mathrm{Cl} 27.4-47.0)$. LV dysfunction was identified in $23(9.5 \%, 95 \% \mathrm{Cl} 6.4-13.9)$ of initial studies of which 13 patients died within 30 days (56.5\%, 95\% CI 36.8-74.4).

\section{Paired studies}

Comparisons were made between the initial studies and a second study taken at a later time point in the 71 cases where serial studies were recorded. The median interval between the initial and second study 
was 4 days (IQR 3-5). There was no statistical difference in mean score comparing the initial and later study (mean 9.0 SD 3.6 vs 9.3 SD 3.8, p value $=0.6$ ). The trajectory of lung severity scores between scans is provided in Fig. 5A. Progression of lung severity score was associated with a small but statistically significant increase in 30-day mortality in a model controlled for the time interval between studies (adjusted OR 1.2 95\% Cl 1.0-1.5, p value $=<0.05$ )

\section{Discussion}

This service evaluation is the first national study of its kind at the time of publication. It included patients admitted with respiratory failure during the COVID-19 pandemic from 12 different UK trusts. Patient demographics from this cohort closely matched ICNARC data ${ }^{21}$, indicating that we studied a generalizable sample.

In our evaluation, combined LUS and FUSIC Heart were performed in $89.5 \%$ of total patients enrolled. This indicates that, even in severely critically ill COVID-19 patients, combined LUS and FUSIC Heart were deliverable alongside the high intensity ICU clinical workload across the trusts.

However, there was a mean time to scan of 2 days from ICU admission, and only $39.5 \%$ of patients received two or more scans. Serial scan data in our study and other papers have shown increased mortality with worsening lung scores ${ }^{22}$. It could be argued, therefore, that there remains suboptimal provision for serial ICU PoCUS services across the trusts involved and potentially nationwide.

The correlation between 12-point and 6-point scores supports the role of the 6-point LUS protocol in ICU patients ${ }^{23},{ }^{24}$. The PosteroLateral Alveolar and/or Pleural Syndrome (PLAPS) is easily achievable and provides good discrimination from the anterior views $25,26,27,28$. Clearly this is beneficial as movement of COVID-19 patients for posterior views requires additional manpower, can be time-consuming and can cause instability.

Typical LUS findings have a good correlation with COVID-19 PCR positivity. This has been well studied and published previously $29,30,31,32,33,34$. Preserved A-lines in all 6 zones made the diagnosis of COVID-19 related respiratory failure extremely unlikely ${ }^{35}$. Alveolar-interstitial syndrome associated with a normal LV function indicates a non-cardiogenic cause for respiratory failure.

LUS was able to identify those who had a higher 30-day mortality, particularly if the total score was $>8$ or there was a high score in the PLAPS zones ${ }^{36}$. The incidence of RV dysfunction was more prevalent in our study in those with higher LUS scores (indicating more extensive COVID lung involvement) ${ }^{37,38}$. This study has demonstrated worse outcomes in patients with RV dysfunction. This association has been extensively shown in ICU patients with RV dysfunction in the context of ARDS and COVID-19 9,39,40,41,42,43 . Lazzeri et al noted LUS score and right:left ventricle ratio (representing RV dilatation) were both independent predictors of ICU mortality in their observational study of 47 ICU patients ${ }^{17}$. 
Most clinical guidelines used fraction of inspired oxygen concentration (FiO2), oxygen saturations and respiratory rate as parameters to identify patients who would benefit from critical care referral ${ }^{44}$. This study suggests that combined LUS score and RV assessment may aid in identifying those patients in a worse prognostic group. Patients with high LUS scores and RV dysfunction may benefit from earlier ICU admission and closer monitoring. However, as this was a service evaluation study, formal research studies will need to be done to assess this further.

$65 \%$ of patients who had POCUS scans had a subsequent change in ICU management. Management strategies were adapted from literature ${ }^{45}$ and utilised on a recommendation basis from clinical advisory groups. Many of these guidelines included the need for LUS and FUSIC Heart to aid decision making ${ }^{46}$. These included optimal PEEP strategies based on lung compliance and right heart function, decision to prone and haemodynamic management strategies aimed at reducing pulmonary hypertension or augmenting the systolic RV function.

A systematic review of PoCUS use in patients with undifferentiated shock ${ }^{47}$ documented two ICU based studies where focused echocardiography resulted in a change in patient management in $41-51 \%$ of patients ${ }^{48,49}$. These studies focused primarily on shock management as an end point. COVID-19 pathophysiology is complex, multi-system, and the potential interventions are numerous and interdependent. This may explain why our percentage of intervention was higher. However, this study did not measure whether interventions led to changes in outcome. There is inherent bias due to this study being performed by enthusiastic PoCUS practitioners and so caution must be taken when interpreting this observation.

Given the association between LUS score $>8$, RV dysfunction and outcome, acquiring this information becomes important. The utility of this information is clear: triaging to a clinical area of higher monitoring, instigating pre-emptive management strategies or altering existing therapies are all possible.

The combination of these two imaging modalities, LUS and FUSIC Heart, in COVID-19 patients at ICU admission (or ideally before) provides dynamic clinical information and informs the complex interactions between the heart and lungs and the effects, if any, of therapeutic intervention. It is therefore crucial to the overall management of this condition.

\section{Limitations}

Service evaluation methodology provides insight into how LUS and FUSIC Heart are utilised in the delivery of routine care at the expense of a defined study protocol which ensures consistency in approach. Based on the observational methodology, the results should be interpreted within the constraints and limitations of a service evaluation study.

There was significant variation in the time to first imaging study and intervals between studies. Including scans performed in the initial 7 days from ICU admission introduces a degree of survivor bias. The use of 
a semi-quantitative score may be an oversimplification of a fundamentally subjective assessment. The salient information which dictated changes may not have been captured within the ordinal scale used.

This is an evaluation of a service that for most was set up de novo to meet the needs of the pandemic. This may explain some of the discrepancies in the results. It seems less likely that a patient admitted to the ICU with severe respiratory failure due to COVID-19 pneumonitis had a lung score of zero, which may reflect issues with PoCUS sonographers' training or reporting.

It is important to note that RV assessment is more challenging than first appreciated. If the left ventricle is hyperdynamic, one may expect the right to be in a similar state. The fact that the right merely appeared to be 'normally' functioning may actually represent dysfunction at this stage in context. The basic level of training has enabled a larger number of patients to have an assessment scan. However, the trade-off is that subtle echo signs such as apical RV dilatation or early systolic dysfunction may have not been appreciated as this would have required an advanced skill set not reliably available on ICUs.

This raises questions around 'quantity versus quality' - is it better to provide a screening service where most patients have a scan or is it desirable to have a detailed analysis in a few, sicker patients? This question is beyond the scope of this study and requires further investigation to understand the optimum way to organise a PoCUS service.

\section{Conclusions}

Our service evaluation suggests that serial, combined LUS and FUSIC Heart can perform an important clinical role in identification of COVID-19, risk-stratification of patients, monitoring disease progress and guiding interventions on ICU. There was a significant association between lung ultrasound score, RV dysfunction and mortality in this study. Further research studies need to be done to further identify the role of LUS and FUSIC Heart in COVID-19 ICU patients.

\section{List Of Abbreviations: (In Order Of Appearance)}

Lung Ultrasound (LUS)

Focused UltraSound for Intensive Care heart (FUSIC)

Focused Intensive Care Echocardiography (FICE)

United Kingdom (UK)

Intensive Care Unit (ICU)

Right Ventricular (RV)

Coronavirus Disease 2019 (COVID-19) 
Point of Care UltraSound (PoCUS)

High Dependency Units (HDU)

Critical Care Echocardiography (CCE)

Left Ventricular (LV)

Acute Respiratory Distress Syndrome (ARDS),

Positive End-Expiratory Pressure (PEEP)

Focused Acute Medicine Ultrasound (FAMUS)

Research Electronic Data Capture (REDCap)

Invasive Mechanical Ventilation (IMV)

Extravascular Lung Water (EVLW)

PosteroLateral Alveolar and/or Pleural Syndrome (PLAPS)

Fraction of Inspired Oxygen concentration (FiO2)

\section{Declarations}

\section{Ethical Approval and Consent to participation}

All participating sites registered the evaluation locally and obtained permission to share anonymised data by the relevant Caldicott Guardian. Data were anonymised locally prior to transfer to the central database (hosted at the University of Birmingham using Research Electronic Data Capture (REDCap) software ${ }^{50}$ ) and identified using a unique study identification number. The study had R\&I approval locally (East Kent Hospitals University NHS Foundation Trust); R\&l reference number: 2021/GAP/13.

\section{Consent for publication}

Local consent sourced, prior to anonymisation, in association with the relevant Caldicott guardian.

\section{Availability of Supporting Data:}

The datasets used during the critical care study are available from the corresponding author at reasonable request.

\section{Competing Interests}

The authors declare that they have no competing interests. 


\section{Funding}

This research received no specific grant from any funding agency in the public, commercial, or not-forprofit sectors.

\section{Author's Contributions}

All authors contributed to study design, implementation and data collection at their local centres.

$P P, J P L, J A$ and TK were the main contributors in writing this paper.

TK was the primary contributor in data analysis.

All authors read and approved the final manuscript.

\section{Acknowledgements:}

We would like to thank all of the members of participating hospital's PoCUS teams without whose hard work the results of the study could not have been acquired.

\section{References}

1. Features of 20133 UK patients in hospital with covid-19 using the ISARIC WHO Clinical Characterisation Protocol: prospective observational cohort study. AB Docherty et al. BMJ 2020;369:m1985

2. Laursen CB, Sloth E, Lassen AT, Christensen Rd, Lambrechtsen J, Madsen PH, Henriksen DP, Davidsen JR, Rasmussen F. Point-of-care ultrasonography in patients admitted with respiratory symptoms: a single-blind, randomised controlled trial. Lancet Respir Med. 2014 Aug;2(8):638-46. doi: 10.1016/S2213-2600(14)70135-3. Epub 2014 Jul 3. PMID: 24998674.

3. Hussain A. et al. Multi-organ point-of-care ultrasound for COVID-19 (PoCUS4COVID): international expert consensus. Crit Care. 2020 Dec 24;24(1):702. doi: 10.1186/s13054-020-03369-5. PMID: 33357240

4. Lichtenstein D, Goldstein I, Mourgeon E, Cluzel P, Grenier P, Rouby JJ. Comparative diagnostic performances of auscultation, chest radiography, and lung ultrasonography in acute respiratory distress syndrome. Anesthesiology. 2004;100(1):9-15

5. Laursen CB, Sloth E, Lambrechtsen J, Lassen AT, Madsen PH, Henriksen DP, Davidsen JR, Rasmussen F. Focused sonography of the heart, lungs, and deep veins identifies missed lifethreatening conditions in admitted patients with acute respiratory symptoms. Chest. 2013 Dec;144(6):1868-1875. doi: 10.1378/chest.13-0882. PMID: 23948720.

6. Lichtenstein, D.A. 'Lung ultrasound in the critically ill.' Ann. Intensive Care 4, 1 (2014). https://doi.org/10.1186/2110-5820-4-1 
7. Vieillard-Baron A, Millington SJ, Sanfilippo F, Chew M, Diaz-Gomez J, McLean A, Pinsky MR, Pulido J, Mayo P, Fletcher N. A decade of progress in critical care echocardiography: a narrative review. Intensive Care Med. 2019 Jun;45(6):770-788. doi: 10.1007/s00134-019-05604-2. Epub 2019 Mar 25. Erratum in: Intensive Care Med. 2019 Apr 15;: PMID: 30911808.

8. Johnson BK, Tierney DM, Rosborough TK, Harris KM, Newell MC. Internal medicine point-of-care ultrasound assessment of left ventricular function correlates with formal echocardiography. J Clin Ultrasound. 2016 Feb;44(2):92-9. doi: 10.1002/jcu.22272. Epub 2015 Jul 14. PMID: 26179460.

9. Boissier, F., Katsahian, S., Razazi, K. et al. 'Prevalence and prognosis of cor pulmonale during protective ventilation for acute respiratory distress syndrome'. Intensive Care Med 39, 1725-1733 (2013). https://doi.org/10.1007/s00134-013-2941-9

10. Repessé X., Charron C., Vieillard-Baron A. (2012) Right ventricular failure in ALI and ARDS. Minerva Anestesiol 78: 941-948.

11. Ranieri VM, Rubenfeld GD, Thompson BT, Ferguson ND, Caldwell E, Fan E, Camporota L, Slutsky AS: 'Acute respiratory distress syndrome: the Berlin Definition. ARDS Definition Task Force', JAMA. 2012 Jun 20; 307(23):2526-33.

12. Peng QY, Wang XT, Zhang LN; Chinese Critical Care Ultrasound Study Group (CCUSG). 'Findings of lung ultrasonography of novel corona virus pneumonia during the 2019-2020 epidemic'. Intensive Care Med. 2020 May;46(5):849-850. doi: 10.1007/s00134-020-05996-6. Epub 2020 Mar 12. PMID: 32166346; PMCID: PMC7080149.

13. Buonsenso D, Piano A, Raffaelli F, et al. Point-of-Care Lung Ultrasound findings in novel coronavirus disease-19 pnemoniae: a case report and potential applications during COVID-19 outbreak. Eur Rev Med Pharmacol Sci. 2020; 24(5): 2776-80.

14. Luciano Gattinoni, Davide Chiumello, Pietro Caironi, Mattia Busana, Federica Romitti, Luca Brazzi, Luigi Camporota COVID-19 pneumonia: different respiratory treatments for different phenotypes? Intensive Care Med. 2020; 46(6): 1099-1102. Published online 2020 Apr 14. doi: 10.1007/s00134020-06033-2 PMCID:PMC7154064

15. Volpicelli, G., Lamorte, A., \& Villén, T. (2020). What's new in lung ultrasound during the COVID-19 pandemic. Intensive care medicine, 46(7), 1445-1448. https://doi.org/10.1007/s00134-020-06048-9

16. Gattinoni L, Chiumello D, Caironi P, Busana M, Romitti F, Brazzi L, Camporota L. COVID-19 pneumonia: different respiratory treatments for different phenotypes? Intensive Care Med. 2020 Jun;46(6):1099-1102. doi: 10.1007/s00134-020-06033-2. Epub 2020 Apr 14. PMID: 32291463; PMCID: PMC7154064.

17. Lazzeri C, Bonizzoli M, Batacchi S, Socci F, Matucci-Cerinic M, Peris A. Combined lung and cardiac ultrasound in COVID-related acute respiratory distress syndrome [published online ahead of print, 2021 Mar 11]. Intern Emerg Med. 2021;1-7. doi:10.1007/s11739-021-02646-7

18. Zhang L, Wang B, Zhou J, Kirkpatrick J, Xie M, Johri AM. Bedside Focused Cardiac Ultrasound in COVID-19 from the Wuhan Epicenter: The Role of Cardiac Point-of-Care Ultrasound, Limited 
Transthoracic Echocardiography, and Critical Care Echocardiography. J Am Soc Echocardiogr. 2020;33(6):676-682. doi:10.1016/j.echo.2020.04.004

19. Mahmoud-Elsayed HM, Moody WE, Bradlow WM, Khan-Kheil AM, Senior J, Hudsmith LE, Steeds RP. Echocardiographic Findings in Patients With COVID-19 Pneumonia. Can J Cardiol. 2020 Aug;36(8):1203-1207. doi: 10.1016/j.cjca.2020.05.030. Epub 2020 May 28. PMID: 32474111; PMCID: PMC7255734.

20. J. Cormack et al.'Time to establish pillars in point-of-care ultrasound Carolynne' First published: 19 February 2019 https://doi.org/10.1002/ajum.12126

21. Richards-Belle A et al. 'COVID-19 in critical care: epidemiology of the first epidemic wave across England, Wales and Northern Ireland'. Intensive Care Med. 2020 Nov;46(11):2035-2047. doi: 10.1007/s00134-020-06267-0. Epub 2020 Oct 9. PMID: 33034689

22. Lichter, Y., Topilsky, Y., Taieb, P. et al. Lung ultrasound predicts clinical course and outcomes in COVID-19 patients. Intensive Care Med 46, 1873-1883 (2020). https://doi.org/10.1007/s00134-02006212-1

23. Juvel Mabao Taculod, Kay Choong See, Juliet Sahagun, Yi Lin Tan, Venetia Ong 'Comparison of 6Point vs. 12-Point Lung Ultrasound for the Diagnosis of Bilateral Lung Infiltrates in ARDS' Respiratory Care Oct 2018, 63 (Suppl 10) 3015557;

24. Guang Song, Wei Qiao, Xin Wang, Xiaona Yu. 'Association of Lung Ultrasound Score with Mortality and Severity of COVID-19: A Meta-Analysis and Trial Sequential Analysis' International Journal of Infectious Diseases, Volume 108, 2021, Pages 603-609, ISSN 1201-9712, https://doi.org/10.1016/j.ijid.2021.06.026.

25. H. Xue et al. M-BLUE protocol for coronavirus disease-19 (COVID-19) patients: interobserver variability and correlation with disease severity CLinical Radiology 2021Volume 76 Issue 5 p379-383

26. Mark E. Haaksma, Micah L. A. Heldeweg, Jorge E. Lopez Matta, Jasper M. Smit, Jessica D. van Trigt, Jip S. Nooitgedacht, Carlos V. Elzo Kraemer, Armand R.J. Girbes, LeoHeunks, David J. van Westerloo, Pieter R. Tuinman. medRxiv Lung BMJ Yale. 'Ultrasound findings in patients with novel SARS-CoV2' 2020.05.18.20105775; https://doi.org/10.1101/2020.05.18.20105775

27. Soldati, G., Smargiassi, A., Inchingolo, R., Buonsenso, D., Perrone, T., Briganti, D.F., Perlini, S., Torri, E., Mariani, A., Mossolani, E.E., Tursi, F., Mento, F. and Demi, L. (2020), Is There a Role for Lung Ultrasound During the COVID-19 Pandemic?. J Ultrasound Med, 39: 1459-1462. https://doi.org/10.1002/jum.15284

28. Lerchbaumer, M.H., Lauryn, J.H., Bachmann, U. et al. Point-of-care lung ultrasound in COVID-19 patients: inter- and intra-observer agreement in a prospective observational study. Sci Rep 11, 10678 (2021). https://doi.org/10.1038/s41598-021-90153-2

29. Zieleskiewicz L, Markarian T, Lopez A, et al. Comparative study of lung ultrasound and chest computed tomography scan in the assessment of severity of confirmed COVID-19 pneumonia. Intensive Care Med. 2020;46(9):1707-1713. doi:10.1007/s00134-020-06186-0 
30. Ji, L., Cao, C., Gao, Y. et al. Prognostic value of bedside lung ultrasound score in patients with COVID19. Crit Care 24, 700 (2020).

31. Lichter Y, Topilsky Y, Taieb P, Banai A, Hochstadt A, Merdler I, Gal Oz A, Vine J, Goren O, Cohen B, Sapir O, Granot Y, Mann T, Friedman S, Angel Y, Adi N, Laufer-Perl M, Ingbir M, Arbel Y, Matot I, Szekely Y. Lung ultrasound predicts clinical course and outcomes in COVID-19 patients. Intensive Care Med. 2020 Oct;46(10):1873-1883. doi: 10.1007/s00134-020-06212-1. Epub 2020 Aug 28. Erratum in: Intensive Care Med. 2020 Sep 25;: PMID: 32860069; PMCID: PMC7454549.

32. Peris, A., Zagli, G., Barbani, F., Tutino, L., Biondi, S., Di Valvasone, S., Batacchi, S., Bonizzoli, M., Spina, R., Miniati, M., Pappagallo, S., Giovannini, V. and Gensini, G.F. (2010), The value of lung ultrasound monitoring in H1N1 acute respiratory distress syndrome. Anaesthesia, 65: 294-297.

33. Kulkarni S, Down B, Jha S. Point-of-care lung ultrasound in intensive care during the COVID-19 pandemic. Clin Radiol. 2020;75(9):710.e1-710.e4. doi:10.1016/j.crad.2020.05.001

34. Lieveld AWE, Kok B, Azijli K, Schuit FH, van de Ven PM, de Korte CL, Nijveldt R, van den Heuvel FMA, Teunissen BP, Hoefsloot W, Nanayakkara PWB, Bosch FH. Assessing COVID-19 pneumonia-Clinical extension and risk with point-of-care ultrasound: A multicenter, prospective, observational study. J Am Coll Emerg Physicians Open. 2021 May 1;2(3):e12429. doi: 10.1002/emp2.12429. PMID: 33969350; PMCID: PMC8087918.

35. Gil-Rodrigo A, Llorens P, Martínez-Buendía C, Luque-Hernández MJ, Espinosa B, Ramos-Rincón JM. Diagnostic yield of point-of-care ultrasound imaging of the lung in patients with COVID-19. Emergencias. 2020 Sep;32(5):340-344. English, Spanish. PMID: 33006834.

36. Bonadia N, Carnicelli A, Piano A, Buonsenso D, Gilardi E, Kadhim C, Torelli E, Petrucci M, Di Maurizio L, Biasucci DG, Fuorlo M, Forte E, Zaccaria R, Franceschi F. Lung Ultrasound Findings Are Associated with Mortality and Need for Intensive Care Admission in COVID-19 Patients Evaluated in the Emergency Department. Ultrasound Med Biol. 2020 Nov;46(11):2927-2937. doi:

10.1016/j.ultrasmedbio.2020.07.005. Epub 2020 Jul 15. PMID: 32798003; PMCID: PMC7362856.

37. Zhao Z, Jiang $L$, Xi X, et al. Prognostic value of extravascular lung water assessed with lung ultrasound score by chest sonography in patients with acute respiratory distress syndrome. BMC Pulm Med. 2015;15:98. Published 2015 Aug 23. doi:10.1186/s12890-015-0091-2

38. Frassi F, Gargani L, Tesorio P, Raciti M, Mottola G, Picano E. Prognostic value of extravascular lung water assessed with ultrasound lung comets by chest sonography in patients with dyspnea and/or chest pain. J Card Fail. 2007 Dec;13(10):830-5. doi: 10.1016/j.cardfail.2007.07.003. PMID: 18068616.

39. The Right Ventricle in ARDS. Zochios V, Parhar K, Tunnicliffe W, Roscoe A, Gao F. Chest. 2017 Jul;152(1):181-193. doi: 10.1016/j.chest.2017.02.019. Epub 2017 Mar 4. PMID: 28267435

40. Lazzeri C, Bonizzoli M, Batacchi S, Peris A. Echocardiographic assessment of the right ventricle in COVID -related acute respiratory syndrome. Intern Emerg Med. 2021;16(1):1-5. doi:10.1007/s11739020-02494-x 
41. Martha JW, Pranata R, Wibowo A, Lim MA. Tricuspid annular plane systolic excursion (TAPSE) measured by echocardiography and mortality in COVID-19: A systematic review and meta-analysis. Int J Infect Dis. 2021 Apr;105:351-356. doi: 10.1016/j.ijid.2021.02.029. Epub 2021 Feb 11. PMID: 33582370; PMCID: PMC7877816

42. Szekely Y, Lichter Y, Taieb P, Banai A, Hochstadt A, Merdler I, Gal Oz A, Rothschild E, Baruch G, Peri Y et al (2020) Spectrum of cardiac manifestations in COVID-19: a systematic echocardiographic study. Circulation 142(4):342-353

43. Deng Q, Hu B, Zhang Y, Wang H, Zhou X, Hu W et al (2020) Suspected myocardial injury in patients with COVID-19: evidence from front-line clinical observation in Wuhan. China Int J Cardiol 311:115121

44. Knight S R, Ho A, Pius R, Buchan I, Carson G, Drake T M et al. Risk stratification of patients admitted to hospital with covid-19 using the ISARIC WHO Clinical Characterisation Protocol: development and validation of the 4C Mortality Score BMJ 2020; 370 :m3339 doi:10.1136/bmj.m3339

45. Lazzeri C, Cianchi G, Bonizzoli M, Batacchi S, Peris A, Gensini GF. The potential role and limitations of echocardiography in acute respiratory distress syndrome. Ther Adv Respir Dis. 2016;10(2):136148. doi:10.1177/1753465815621251

46. Mozzini C, Di Dio Perna M, Pesce G, Garbin U, Fratta Pasini AM, Ticinesi A, Nouvenne A, Meschi T, Casadei A, Soresi M, Cominacini L. Lung ultrasound in internal medicine efficiently drives the management of patients with heart failure and speeds up the discharge time. Intern Emerg Med. 2018 Jan;13(1):27-33. doi: 10.1007/s11739-017-1738-1. Epub 2017 Aug 12. PMID: 28803375.

47. Heiberg, J., El-Ansary, D., Canty, D.J., Royse, A.G. and Royse, C.F. (2016), Focused echocardiography: a systematic review of diagnostic and clinical decision-making in anaesthesia and critical care. Anaesthesia, 71: 1091-1100. https://doi.org/10.1111/anae.13525

48. Joseph MX, Disney PJ, Da Costa R, Hutchison SJ. Transthoracic echocardiography to identify or exclude cardiac cause of shock. Chest 2004; 126: 1592-7.

49. Stanko LK, Jacobsohn E, Tam JW, De Wet CJ, Avidan M. Transthoracic echocardiography: impact on diagnosis and management in tertiary care intensive care units. Anaesthesia and Intensive Care 2005; 33: 492-6

50. PA Harris, R Taylor, BL Minor, V Elliott, M Fernandez, L O’Neal, L McLeod, G Delacqua, F Delacqua, J Kirby, SN Duda, REDCap Consortium, The REDCap consortium: Building an international community of software partners, J Biomed Inform. 2019 May 9 [doi: 10.1016/j.jbi.2019.103208]

\section{Figures}


A

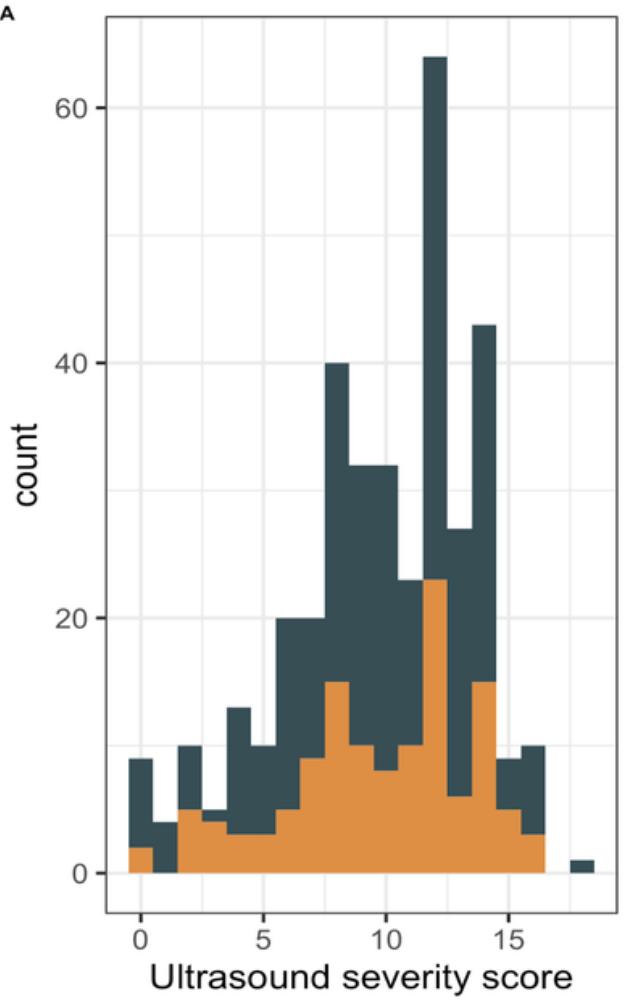

$\leq 7$ days $\square>7$ days
B
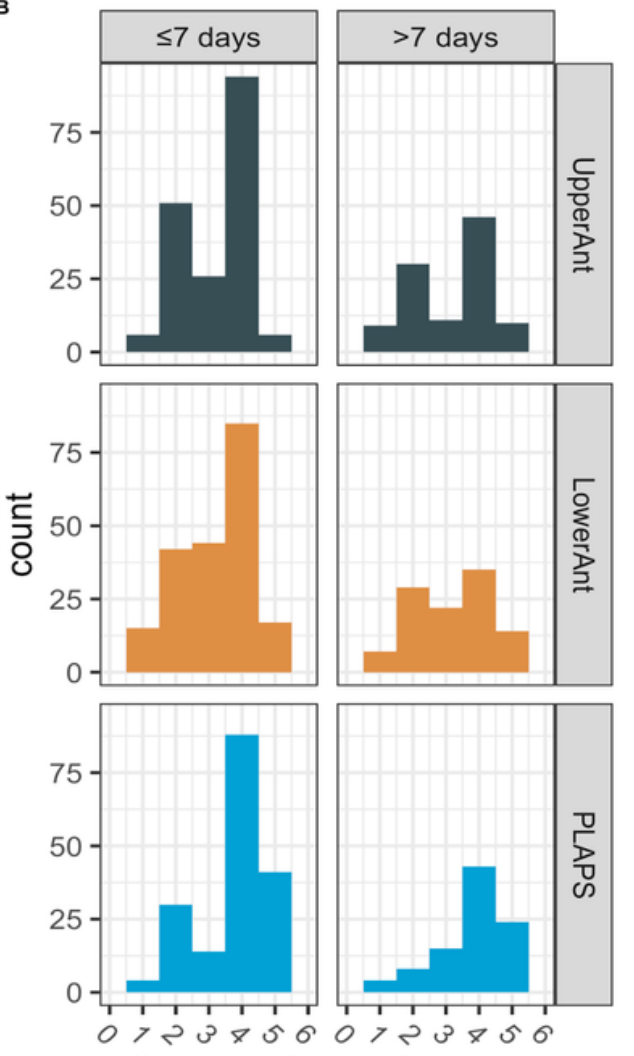

Ultrasound severity score

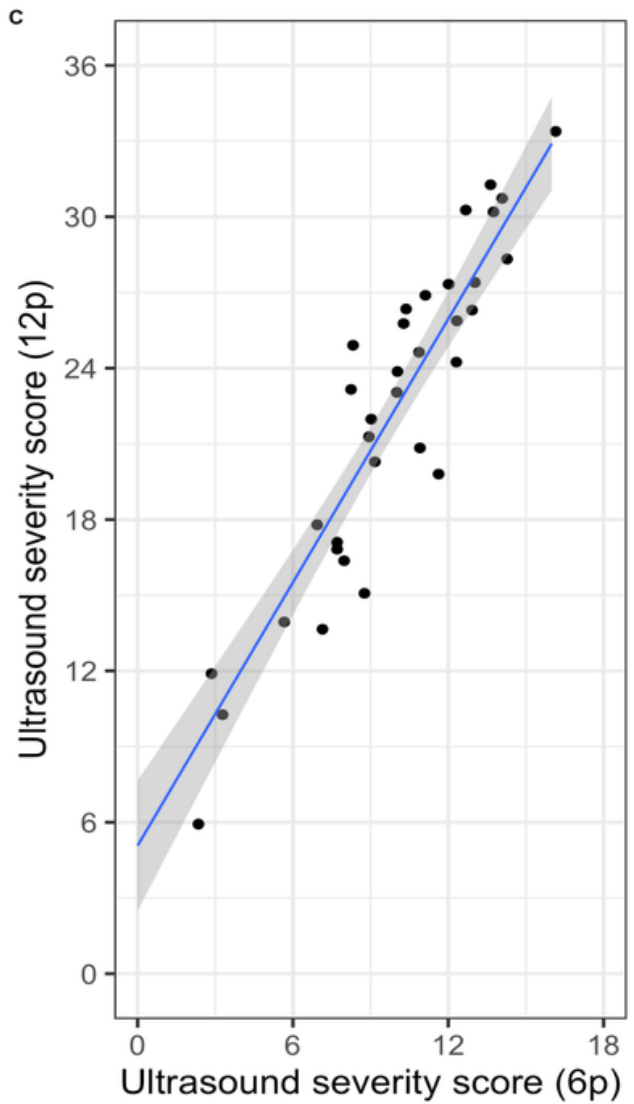

Ultrasound severity score $(6 p)$

\section{Figure 1}

Lung severity scoring A: Distribution of 6-point score stratified by the interval between admission and imaging B: Distribution of scores stratified by anatomical location C: Correlation between 6-point and 12point scan 

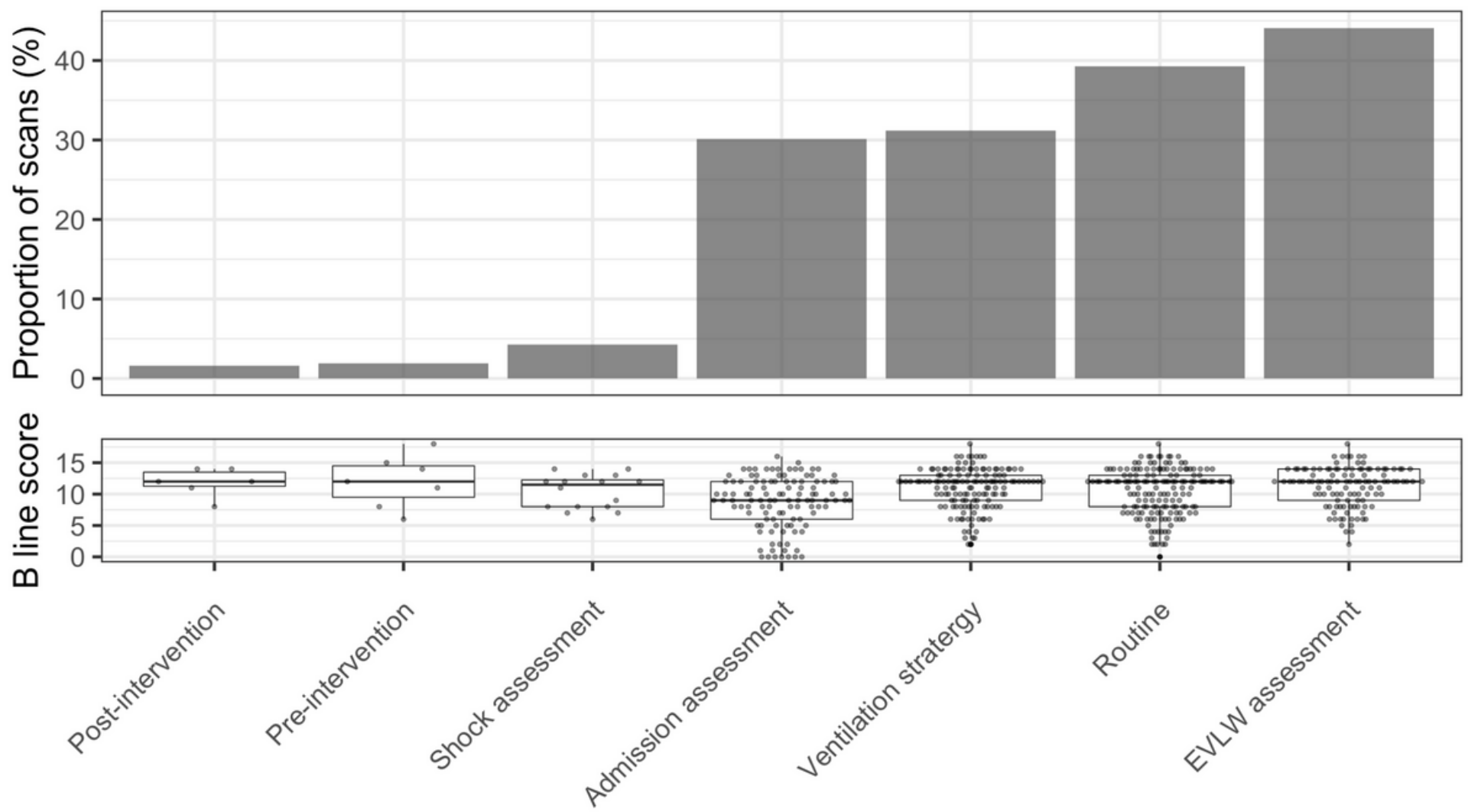

Figure 2

Indication for lung assessment
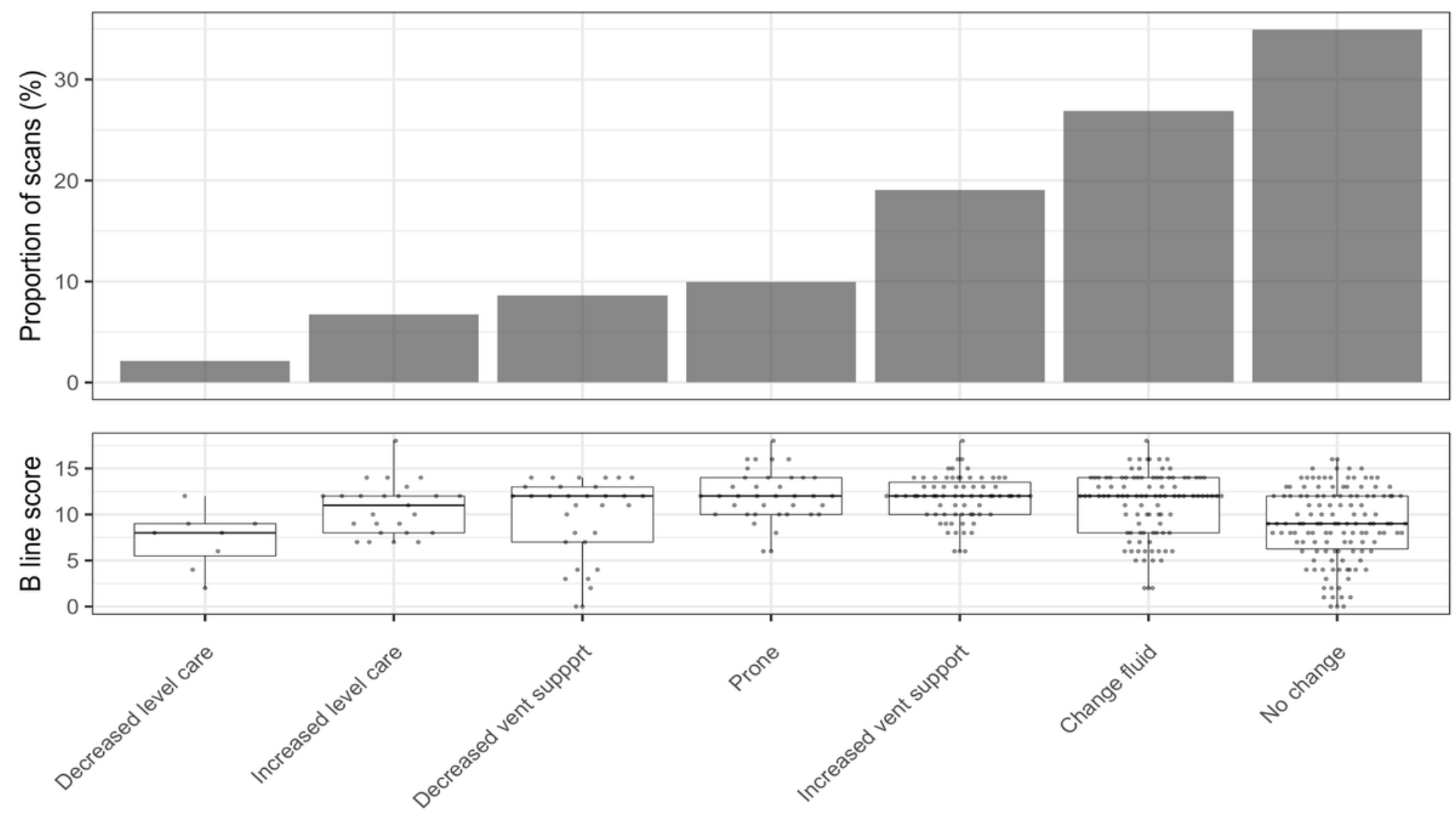
Figure 3

Outcome following PoCUS scan
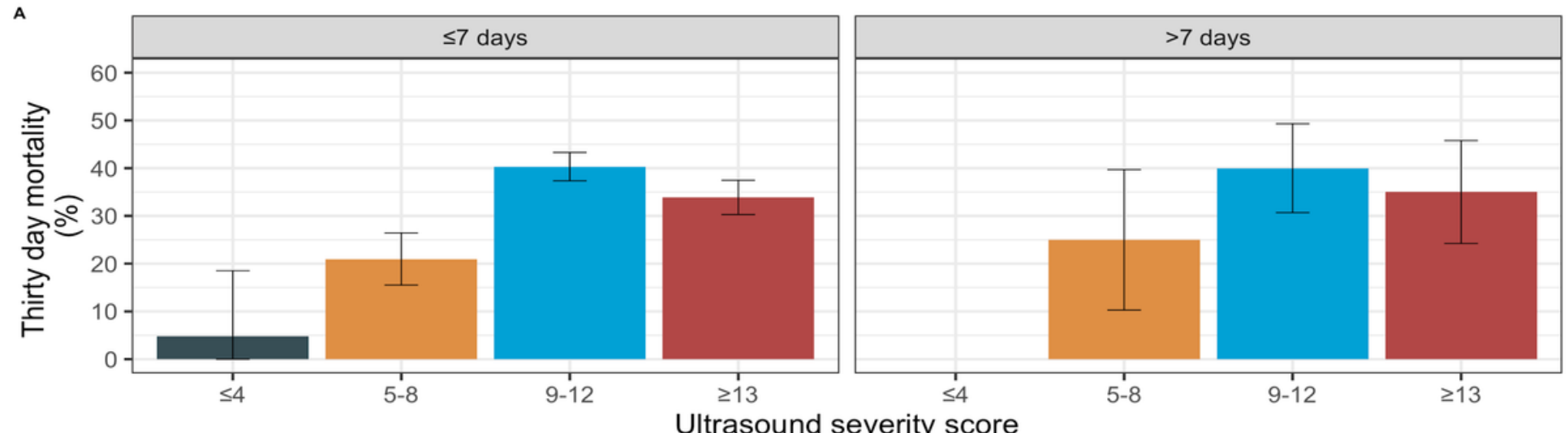

B

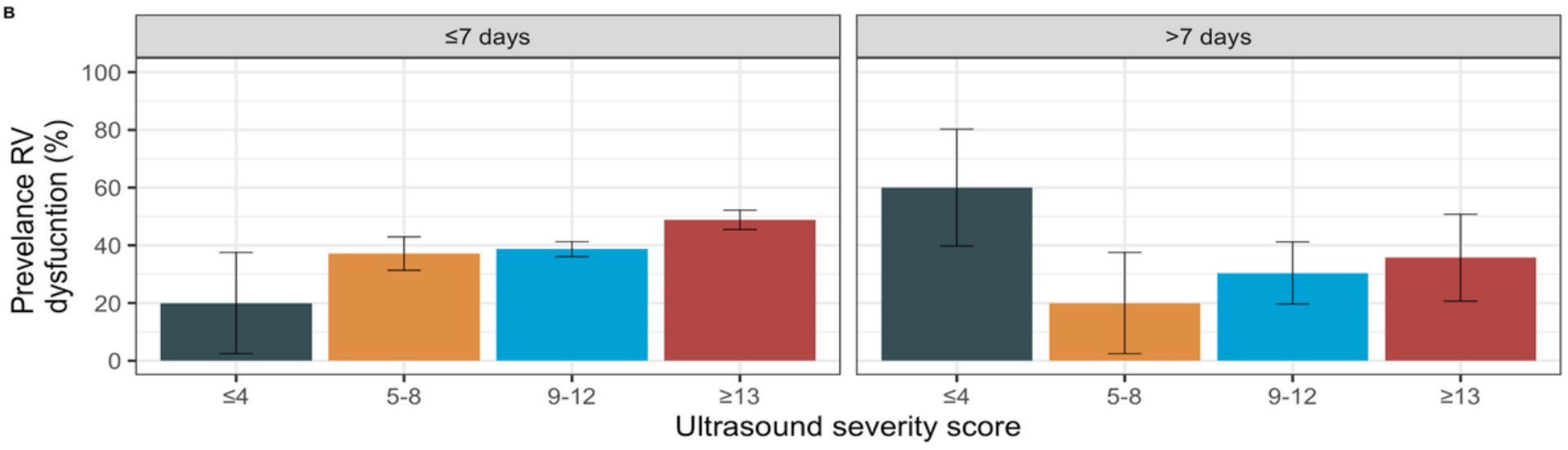

Figure 4

30-day mortality and prevalence of RV dysfunction: A: 30-day mortality stratified by ultrasound severity score and interval between admission and imaging. B: Prevalence of RV dysfunction stratified by ultrasound severity score and interval between admission and imaging. 
A

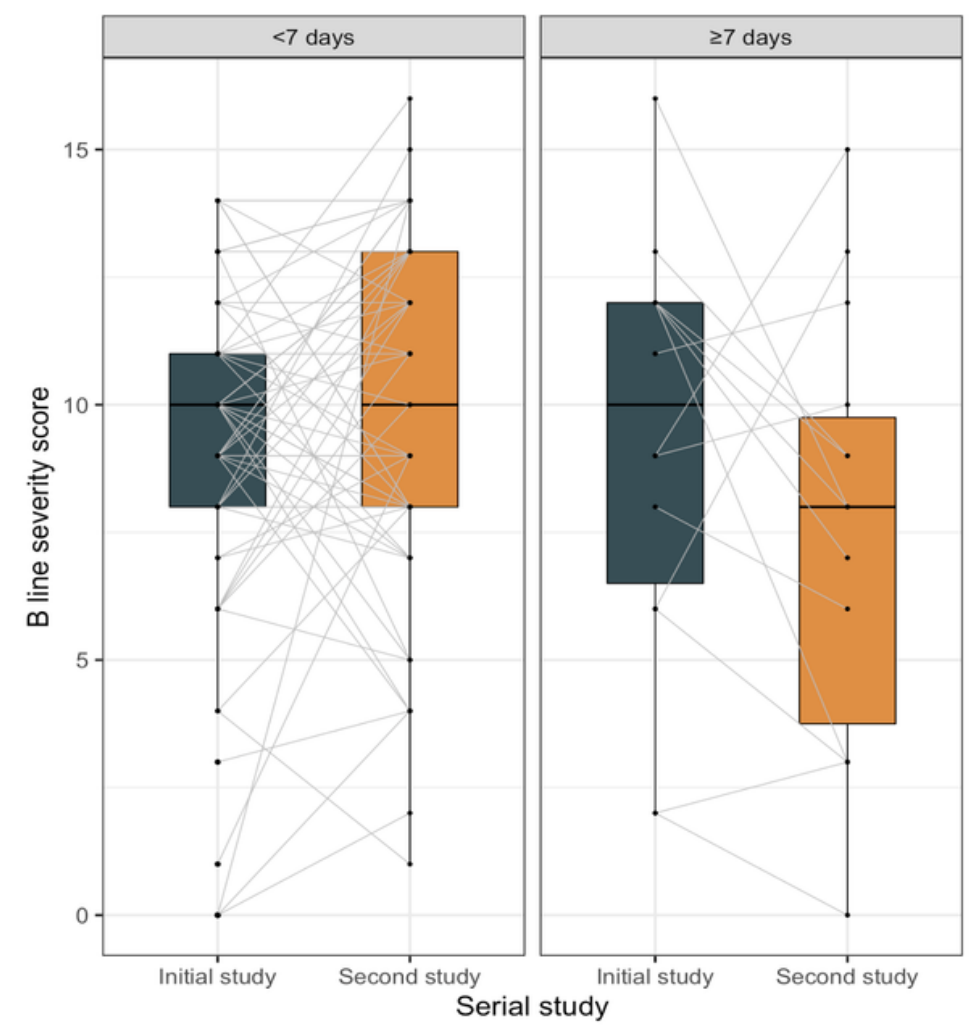

B

30 day mortality $\bullet$ Survived $\bullet$ Died

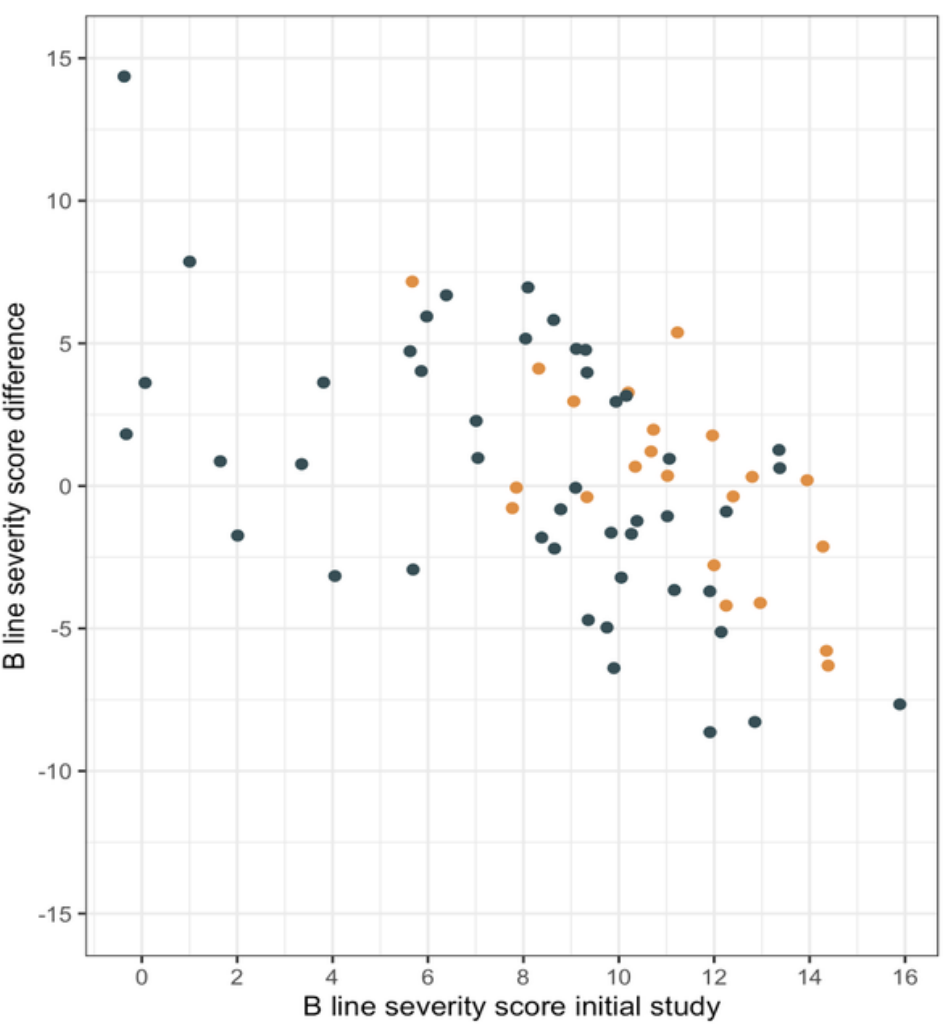

Figure 5

Paired imaging studies: A: Paired ultrasound severity scores B: Initial B line severity score plotted against change in score from the second study.

\section{Supplementary Files}

This is a list of supplementary files associated with this preprint. Click to download.

- Corona.pdf 